\title{
Diferencias por edad en la aceptación de cursos en línea masivos y abiertos (MOOCs)
}

\section{Age differences in the acceptance of Massive Open Online Courses (MOOCs)}

\author{
iD Francisco Rocha; francisco.rochaes@uanl.edu.mx \\ Universidad Autónoma de Nuevo León (México) \\ iD Brenda Cecilia Padilla Rodríguez; brenda.padillardr@uanl.edu.mx \\ Universidad Autónoma de Nuevo León (México) \\ iD Juan Carlos Aguado Franco; juancarlos.aguado@urjc.es \\ Universidad Rey Juan Carlos (España)
}

Resumen

El objetivo de este trabajo fue evaluar la aceptación de los cursos en línea masivos y abiertos (MOOC, por sus siglas en inglés) en relación con la edad y la generación de los usuarios. La muestra comprendió 103 estudiantes de un programa presencial de aprendizaje de inglés, quienes realizaban MOOC como complemento a su formación. Contestaron una versión en español de una escala basada en el modelo de aceptación de tecnología y adaptada a MOOC. Reportaron una percepción positiva hacia los MOOC y describieron sus beneficios. La edad tuvo una relación débil, positiva y significativa con los factores de aceptación: percepciones de utilidad, facilidad de uso y disfrute. Se presentaron diferencias generacionales, pero sólo las de percepción de utilidad fueron significativas.

Palabras clave: aceptación, MOOCs, edad, baby boomers, generación $\mathrm{X}$, millennials

\section{Abstract}

This study aimed to assess the acceptance of massive open online courses (MOOCs), and to analyze its relationship with users' chronological age and generation. The sample was comprised of 103 students enrolled in a face-to-face English language program. They participated in MOOCs as a complement to their course. They answered a Spanish version of a scale based on the technology acceptance model, adapted to MOOCs. Respondents reported having a positive perception towards MOOCs and described benefits related to participating in this type of course. Age had a week, positive and statistically significant relationship with the factors of MOOC acceptance: perceived usefulness, ease of use and enjoyment. While there were generational differences, only the ones related to the perception of usefulness of MOOCs were significant.

Keywords: acceptance, MOOCs, age, baby boomers, generation $X$, millennials 


\section{INTRODUCCIÓN}

Desde su surgimiento, los cursos en línea masivos y abiertos (MOOC, por sus siglas en inglés) han generado interés y controversia entre la comunidad académica. Son intervenciones académicas organizadas, usualmente con fechas de inicio y fin, a las que se puede acceder vía Internet de forma gratuita. Así, miles de estudiantes de todas las edades y de diferentes contextos llegan a enrolarse, participar simultáneamente en este tipo de cursos y conectarse con una comunidad internacional (Shah y Pickard, 2019). Los MOOC representan oportunidades de aprendizaje con un mayor alcance que alternativas tradicionales (Méndez García, 2013; Siemens, 2013). Sólo requieren que los participantes tengan un dispositivo con conexión a internet y una competencia tecnológica básica (Fernández-Ferrer, 2017). Pueden usarse como una herramienta de educación continua o como un complemento a cursos formales, con la ventaja de que los estudiantes pueden revisar los temas de su preferencia a su propio ritmo (Aguado Franco, 2017; Annabi y Wilkins, 2016; Jiménez Cruz, 2017). Tal ha sido su popularidad e impacto que 2012 fue considerado "el año del MOOC" (Pappano, 2012), e incluso hoy se considera un formato exitoso para el aprendizaje en línea (González de la Fuente y Carabantes Alarcón, 2017).

Pese a sus beneficios, los MOOC han sido criticados. Sus detractores argumentan que su carácter innovador se mantiene en lo tecnológico, mas no en lo pedagógico (Armellini y Padilla Rodríguez, 2016). Hay cuestionamientos sobre su calidad educativa, la cual es percibida por algunos estudiantes como menor en comparación con la de cursos tradicionales. Aún hay retos para asegurar que las personas que se inscriben en los cursos son las mismas que completan las evaluaciones (Annabi y Wilkins, 2016; Cole y Timmerman, 2015). Asimismo, cada vez son más los MOOC que requieren un pago para acceder a los materiales y actividades, limitando el carácter abierto de este tipo de cursos (Méndez García, 2013). Finalmente, si bien originalmente se esperaba que los MOOC facilitaran la universalización de la educación, esto no se está alcanzando. En promedio, sólo el 15\% de quienes se inscriben en un MOOC lo terminan (Jordan, 2015). La mayoría de los participantes cuentan ya con estudios universitarios y provienen de países desarrollados; no son personas con dificultades de acceso a oportunidades estructuradas de aprendizaje (Poy y Gonzales-Aguilar, 2014).

\section{Aceptación de MOOC}

Aunque la aceptación de los MOOC por parte de académicos y facilitadores sigue en debate (Armellini y Padilla Rodríguez, 2016), los estudiantes reportan principalmente actitudes positivas. Valoran el fácil acceso a los MOOC, los contenidos actualizados, la flexibilidad, la opción de avanzar a un ritmo personalizado y la experiencia de aprendizaje (Aboshady et al., 2015; Vivar Zurita, Vinader Segura, y Abuín Vences, 2015). Consideran que estos cursos son interesantes, útiles, informativos y disfrutables (Nordin, Norman, y Amin, 2015; Padilla Rodríguez, Bird, y Conole, 2015). Los utilizan para complementar su formación académica y desarrollar sus habilidades laborales (Mulik, Yajnik, y Godse, 2016). Reportan un alto grado de satisfacción 
independientemente si han terminado los MOOC o no (González de la Fuente y Carabantes Alarcón, 2017).

Para estudiar la aceptación y uso de MOOC, el modelo de aceptación de tecnología (TAM, por sus siglas en inglés) presenta una aproximación considerada válida y confiable. Comprende tres factores:

1. Percepción de utilidad - qué tanto una persona cree que mejorará su rendimiento usando una tecnología en particular;

2. Percepción de facilidad de uso - qué tan sencillo es realizar las tareas utilizando cierta tecnología; y

3. Percepción de disfrute - qué tan agradable es percibida la actividad de usar la tecnología (Davis, 1989; Davis, Bagozzi, y Warshaw, 1992).

El TAM se ha probado empíricamente con distintas poblaciones, en diversos entornos y en relación a tecnologías variadas (Marangunić y Granić, 2013; Turner et al., 2010). Además, permite predecir la intención de participar en MOOC (Castaño Garrido, Maiz Olazabalaga, y Garay Ruiz, 2015; Tao, Fu, Wang, Zhang, y Qu, 2019; Wu y Chen, 2017). La percepción de facilidad de uso y utilidad tienen influencia sobre la satisfacción de los estudiantes (Joo, So, y Kim, 2018).

La aceptación de tecnologías del aprendizaje se ve afectada por las habilidades digitales de los individuos y su transferencia a contextos educativos (Gallardo Echenique, 2012). La competencia tecnológica es usualmente mayor a menor edad (Chung et al., 2010; Díaz García, Cebrián Cifuentes y Fuster Palacios, 2016) y permite predecir la intención de utilizar MOOC (Khan et al., 2018). En una investigación donde los MOOC fueron aceptados positivamente, el 91\% de los participantes $(n=1,055)$ se consideraba competente en el uso de las tecnologías de la información (Nordin et al., 2015).

La edad también parece estar relacionada con la aceptación de nuevas herramientas tecnológicas. Por ejemplo, los adultos mayores de 55 años pueden requerir adecuaciones de diseño para aprovechar al máximo algunas herramientas digitales (Angulo Mendoza, Sauvé, y Plante, 2017). Si bien hay cierta evidencia de que las percepciones de facilidad de uso, utilidad e intención de participar no cambian en función de la generación de los usuarios (eg, Chung, Park, Wang, Fulk, y McLaughlin, 2010), un meta-análisis de 144 estudios encontró relaciones negativas y significativas. Las personas más jóvenes mostraban una mayor aceptación. Estos efectos eran bajos o moderados, pero estables a través del tiempo y no resultado de los cohortes. Sin embargo, la relación negativa entre la edad y la percepción de utilidad era influenciada por el tipo de tecnologías. No se mantenía con aquellas que atendían las necesidades sociales y emocionales específicas de los adultos mayores (Hauk, Hüffmeier, y Krumm, 2018).

En los MOOC, personas de todas las edades se benefician. Muchos usuarios son profesionistas que se inscriben y participan como parte de su trayectoria de educación continua o su desarrollo profesional (Castaño Garrido et al., 2015; Padilla Rodríguez et al., 2015). Análisis de los datos 
sociodemográficos de las personas en plataformas de MOOC han revelado una diversidad de perfiles. Por ejemplo, en MiríadaX ( $n=191,608)$ más de la mitad de los usuarios son menores de 34 años y poco más del 15\% es mayor a 45 (Oliver, Hernández-Leo, y Albó, 2015). En HarvardX y MITx la mediana de la edad promedio es de 28 años (Ho et al., 2015). Esto indica que los MOOC atienden las necesidades no sólo de universitarios jóvenes sino también de adultos mayores.

En general, los jóvenes aceptan fácilmente las nuevas tecnologías. Los nacidos en 1982 o después tienden a sentirse fascinados con las herramientas digitales y a incorporarlas en sus prácticas de aprendizaje (Oblinger, 2003). En una investigación de 46 programas intergeneracionales facilitados mediante tecnologías se encontró que los jóvenes de 15 a 24 años se posicionaban más rápidamente en el rol de expertos digitales frente a los adultos de 25 a 54 años y las personas mayores de 55 años (Sánchez, Kaplan, y Bradley, 2015). Esta tendencia también se presenta en docentes; los de menor edad emplean más tecnologías en sus actividades de enseñanza que sus contrapartes de generaciones anteriores (Tapia Cortes, Navarro Rangel, y de la Serna Tuya, 2017).

Comparar la aceptación de MOOC de usuarios de distintas edades y generaciones proporciona información relevante para comprender el fenómeno de los MOOC. El presente trabajo se fundamenta en el TAM para realizar este análisis y revisa también la habilidad tecnológica percibida. Para contribuir a este debate sobre los MOOC y su papel en el escenario educativo, se enfoca en un grupo de estudiantes mexicanos de un programa presencial de inglés quienes cursaron MOOC como complemento a su formación. El objetivo fue evaluar su aceptación de este tipo de cursos, determinando la relación con su edad y las diferencias generacionales.

\section{METODOLOGÍA}

\section{Participantes}

La muestra comprendió 103 estudiantes mexicanos quienes se encontraban inscritos en niveles avanzados de un programa presencial de inglés. Ellos realizaban MOOC como complemento a su formación. Podían elegir la plataforma (MiríadaX, FutureLearn, edX, Coursera u otra) y el curso que mejor correspondiera a sus intereses y preferencias. Contaban con un rango de edad de 18 a 60 años, con una media de 32.5 y una mediana de 29. Fueron divididos de acuerdo a la generación a la que pertenecían (ver Tabla 1). A pesar de que el tamaño de los grupos es dispar, es similar a las proporciones reportadas en plataformas de MOOC como MiríadaX (Oliver et al., 2015), donde el $63 \%$ son millennials; el 20\%, generación X; y una minoría, baby boomers. 
Rocha Estrada, F. J., Padilla Rodríguez, B. C., y Aguado Franco, J. C.

Tabla 1. Participantes por generación

\begin{tabular}{|l|l|l|}
\hline Generación & Descripción & N \\
\hline $\begin{array}{l}\text { Baby boomers } \\
(1946-1967)\end{array}$ & $\begin{array}{l}\text { Adquirieron su nombre debido al gran aumento en las tasas de natalidad } \\
\text { después de la Segunda Guerra Mundial. Se caracterizaron por el uso de aparatos } \\
\text { eléctricos. }\end{array}$ & 17 \\
\hline $\begin{array}{l}\text { Generación X } \\
(1968-1982)\end{array}$ & $\begin{array}{l}\text { Fueron jóvenes que luchaban contra un sistema establecido por la generación } \\
\text { anterior. Comenzaron a utilizar dispositivos electrónicos y vivieron el nacimiento } \\
\text { de internet. }\end{array}$ & 19 \\
\hline $\begin{array}{l}\text { Millennials } \\
(1982-1999)\end{array}$ & $\begin{array}{l}\text { Obtuvieron su nombre gracias a la llegada del nuevo milenio. Crecieron en un } \\
\text { mundo interconectado con las tecnologías de la información y comunicación. }\end{array}$ & 67 \\
\hline
\end{tabular}

Nota. Descripciones basadas en Hayes, Parks, McNeilly y Johnson (2018), y Oblinger (2003)

\section{Instrumentos}

Para evaluar la aceptación de los MOOC, se empleó una escala en español basada en el modelo de aceptación de tecnología (TAM) adaptado a MOOC (Castaño Garrido et al., 2015). Este instrumento cuenta con 12 ítems tipo Likert, con siete opciones de respuestas, que van desde "totalmente en desacuerdo" hasta "totalmente de acuerdo". El TAM cuenta con una consistencia interna satisfactoria y alfas de entre 0.73-0.91 (Castaño Garrido et al., 2015; Davis et al., 1992). Se compone de tres elementos:

- Percepción de utilidad (PU)

- PU1: Creo que la manera de trabajar en el MOOC es provechosa para el aprendizaje.

- PU2: La utilización de vídeo y actividades en línea en el MOOC facilita la comprensión de ciertos aspectos de los temas a aprender.

- PU3: Me parece útil para el aprendizaje interactuar con otras personas a través de internet.

- PU4: El uso de la plataforma del MOOC hace más interesante el aprendizaje.

- PU5: Creo que aprender a través de un MOOC es una buena idea.

- Percepción de facilidad de uso (PF)

- PF1: Creo que la plataforma del MOOC (eg, Coursera, edX, FutureLearn, etc.) es fácil de usar.

- PF2: Aprender a utilizar la plataforma del MOOC es un problema.

- PF3: El manejo de la plataforma del MOOC es claro y comprensible.

- Percepción de disfrute (PD)

- PD1: He disfrutado mientras aprendía en el MOOC. 
- PD2: La pasé bien aprendiendo en el MOOC.

- PD3: Aprender en el MOOC es entretenido.

- PD4: Aprender a través de un MOOC fue aburrido.

Se creó una versión en línea de este instrumento, la cual iniciaba con una explicación del estudio, la confidencialidad de los datos y el carácter voluntario de la participación. Se solicitó la aceptación explícita a ser parte de esta investigación. Al final se incluyó una pregunta tipo Likert de cinco puntos sobre la habilidad percibida en el uso de tecnología, y un espacio abierto para comentarios opcionales.

\section{Procedimiento}

Se contactó a los estudiantes por medio de grupos en Facebook específicos para el programa presencial de inglés al que pertenecían. Se explicaron las características y propósitos de esta investigación, y se resolvieron dudas. Después se publicó un enlace a la versión en línea del instrumento. Se recibieron respuestas durante cinco semanas.

Para conocer la aceptación de los MOOC se obtuvieron las medias y desviaciones estándar de cada subescala del TAM. Asimismo se revisaron los porcentajes de las respuestas sobre la habilidad percibida en el uso de la tecnología.

Para analizar las diferencias en la aceptación de MOOC entre las generaciones, se utilizó la prueba no paramétrica de Kruskal-Wallis. Se calcularon correlaciones de Spearman para evaluar las relaciones entre la edad, los factores de aceptación de los MOOC y la habilidad percibida en el uso de tecnología.

Los comentarios opcionales fueron codificados inductivamente para identificar patrones comunes y temas salientes. Para mantener el anonimato de los participantes, se les asignó un identificador genérico compuesto por una letra correspondiente a la generación (M para millenial, GX para generación X y BB para baby boomer) y un número.

\section{RESULTADOS Y DISCUSIÓN}

Los participantes se inscribieron en distintos $\mathrm{MOOC}$ como complemento a un programa presencial para aprender inglés, como en Aguado Franco (2017) y Jiménez Cruz (2017). Reportaron percepciones predominantemente positivas en cuanto a los tres factores de la aceptación de MOOC: utilidad, facilidad de uso y disfrute (ver Tabla 2). Así como en reportes previos (Aboshady et al., 2015; Castaño Garrido et al., 2015; González de la Fuente y Carabantes Alarcón, 2017; Nordin et al., 2015; Vivar Zurita et al., 2015), se determinó que existe una aceptación hacia los cursos en línea masivos abiertos. 
Tabla 2. Factores de aceptación de MOOC

\begin{tabular}{|c|c|c|c|c|c|c|c|}
\hline \multicolumn{6}{|l|}{ Media por ítem } & \multirow{2}{*}{$\begin{array}{l}\text { Media total } \\
5.97\end{array}$} & \multirow{2}{*}{$\begin{array}{l}\text { Desviación estándar } \\
\pm 1.00\end{array}$} \\
\hline \multirow[t]{2}{*}{ Percepción de utilidad } & PU1 & PU2 & PU3 & PU4 & PU5 & & \\
\hline & 6.04 & 6.30 & 5.83 & 5.77 & 5.89 & & \\
\hline \multirow[t]{2}{*}{ Percepción de facilidad de uso } & PF1 & \multicolumn{2}{|c|}{ PF2 } & PF3 & & \multirow[t]{2}{*}{5.79} & \multirow[t]{2}{*}{ \pm 1.19} \\
\hline & 6.02 & \multicolumn{2}{|c|}{5.59} & \multicolumn{2}{|c|}{5.77} & & \\
\hline \multirow[t]{2}{*}{ Percepción de disfrute } & PD1 & PD2 & $P L$ & 3 & P4 & \multirow[t]{2}{*}{5.85} & \multirow[t]{2}{*}{ \pm 1.08} \\
\hline & 5.85 & 5.94 & 5. & 82 & 5.79 & & \\
\hline
\end{tabular}

Nota. Puntuación máxima $=7$

Todos los factores de la aceptación mostraron correlaciones positivas y significativas (ver Tabla 3). Cuando los participantes perciben los MOOC como fáciles de usar, los consideran más útiles y disfrutan más su experiencia en ellos.

Tabla 3. Relación entre los elementos de la aceptación de MOOC

\begin{tabular}{|c|c|c|c|}
\hline & $\begin{array}{l}\text { Percepción de } \\
\text { utilidad }\end{array}$ & $\begin{array}{l}\text { Percepción de facilidad de } \\
\text { uso }\end{array}$ & $\begin{array}{l}\text { Percepción de } \\
\text { disfrute }\end{array}$ \\
\hline Percepción de utilidad & - & $\begin{array}{l}\text { rho }=.533 \\
p=.000\end{array}$ & $\begin{array}{l}\text { rho }=.735 \\
p=.000\end{array}$ \\
\hline $\begin{array}{l}\text { Percepción de facilidad de } \\
\text { uso }\end{array}$ & $\begin{array}{l}\text { rho }=.533 \\
p=.000\end{array}$ & - & $\begin{array}{l}\text { rho }=.535 \\
p=.000\end{array}$ \\
\hline Percepción de disfrute & $\begin{array}{l}\text { rho }=.735 \\
p=.000\end{array}$ & $\begin{array}{l}\text { rho }=.535 \\
p=.000\end{array}$ & - \\
\hline
\end{tabular}


En el espacio para comentarios opcionales, los participantes describieron beneficios que encuentran al utilizar MOOC:

- "[Estudiar MOOC] es una buena oportunidad para aprender sobre temas de nuestro interés y lo mejor es saber que son de fuentes serias, responsables y además son gratis" [GX26].

- "La flexibilidad de horarios es lo mejor, porque puedes aprender cuando tienes tiempo libre" [M33].

- "Cuando realizas cursos obligados [obligatoriamente] no te resultan nada útiles y el aprendizaje significativo es poco o nulo. Sin embargo al realizar un curso de interés y por forma voluntaria se aprende más..." [M35].

- "Me encanta aprender utilizando todas las herramientas posibles" [BB50].

Podría suponerse intuitivamente que los más jóvenes, quienes nacieron inmersos en una cultura tecnológica (Hayes et al., 2018; Oblinger, 2003), se posicionarían como expertos digitales (Sánchez et al., 2015) y valorarían más los beneficios de los MOOC. No obstante, en este estudio la edad de los participantes y todos los factores de la aceptación de MOOC mostraron relaciones débiles pero positivas y significativas (ver Tabla 4). A mayor edad de los usuarios, se reportaron percepciones más positivas de utilidad, facilidad de uso y disfrute de MOOC.

Tabla 4. Relación entre la edad de los usuarios y los factores de aceptación de MOOC

\begin{tabular}{|l|l|l|l|}
\hline & Percepción de utilidad & Percepción de facilidad de uso & Percepción de disfrute \\
\hline Edad & rho=.371 & rho $=.270$ & rho=.313 \\
$p=.000$ & $p=.006$ & $p=.001$ \\
\hline
\end{tabular}

Al realizar comparaciones por generación, se encontró que todos los factores de aceptación eran positivamente percibidos en todos los grupos. Los baby boomers mostraron una percepción ligeramente más positiva de utilidad, facilidad de uso y disfrute de los MOOC que los millennials y la generación X (ver Figura 1). 


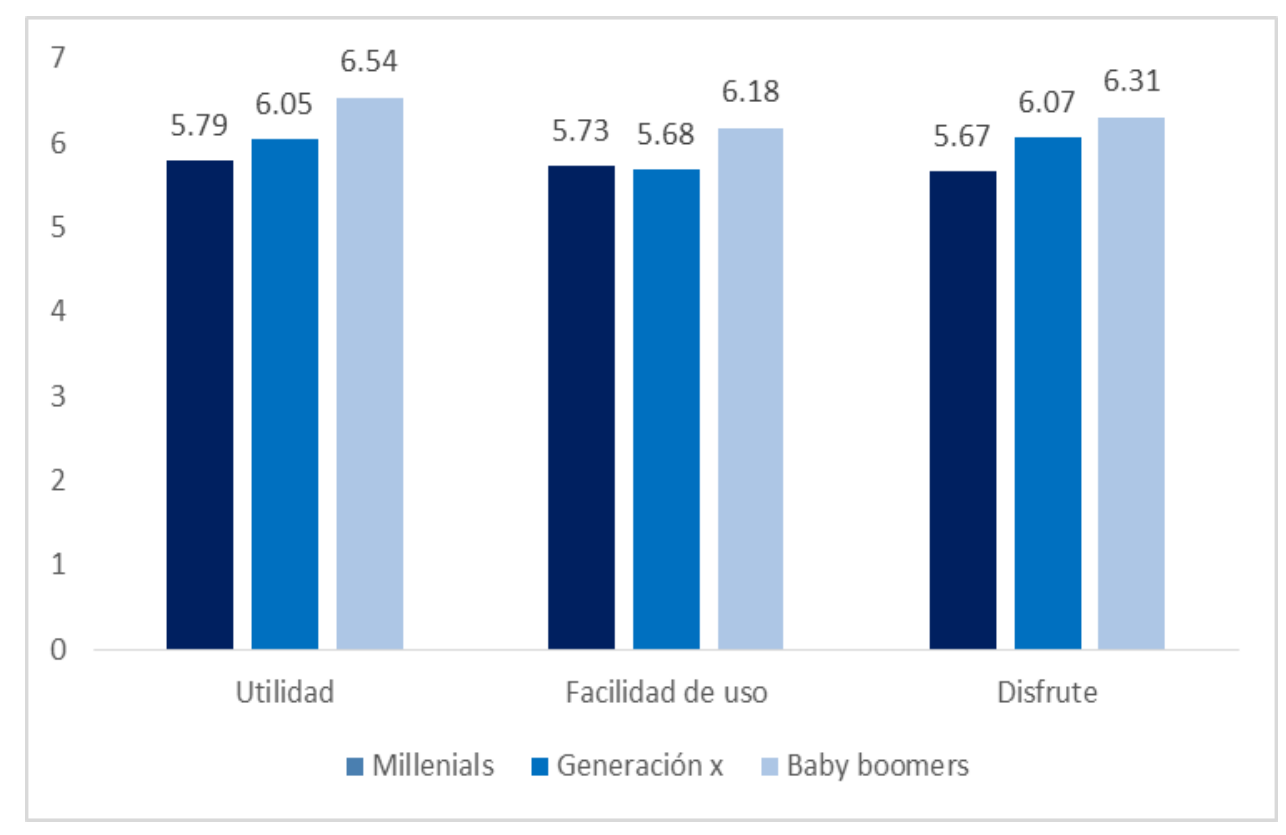

Figura 1. Aceptación de MOOC por generación.

Solo la diferencia de las percepciones de utilidad fue estadísticamente significativa $\left(\chi^{2}=8.309, \mathrm{~g} \mid=2\right.$, $p=0.016)$. Pudiera ser que aunque los jóvenes cuentan con cierta competencia tecnológica, no se consideran capaces de transferirla a un ámbito educativo (Gallardo Echenique, 2012). O quizá los adultos mayores consideran más útiles los MOOC, ya que les representan oportunidades educativas que ya no están a su alcance (y sí lo están para los universitarios) y les permiten actualizar sus conocimientos (Castaño Garrido et al., 2015).

Los estudiantes que cursan $\mathrm{MOOC}$ requieren ser competentes tecnológicamente hablando (Fernández-Ferrer, 2017; Nordin et al., 2015). En concordancia con esto, el 73\% de los participantes percibió su habilidad en el uso de la tecnología como buena o muy buena. Al analizar los datos por generaciones no se mantiene esta tendencia. Más de la mitad de los baby boomers consideró su habilidad como regular o muy mala (ver Figura 2). Una correlación de Spearman mostró que a mayor edad, menor habilidad tecnológica percibida ( $r h o=-.307, p=.002$ ), como en Chung et al. (2010) y Díaz García et al. (2016). 


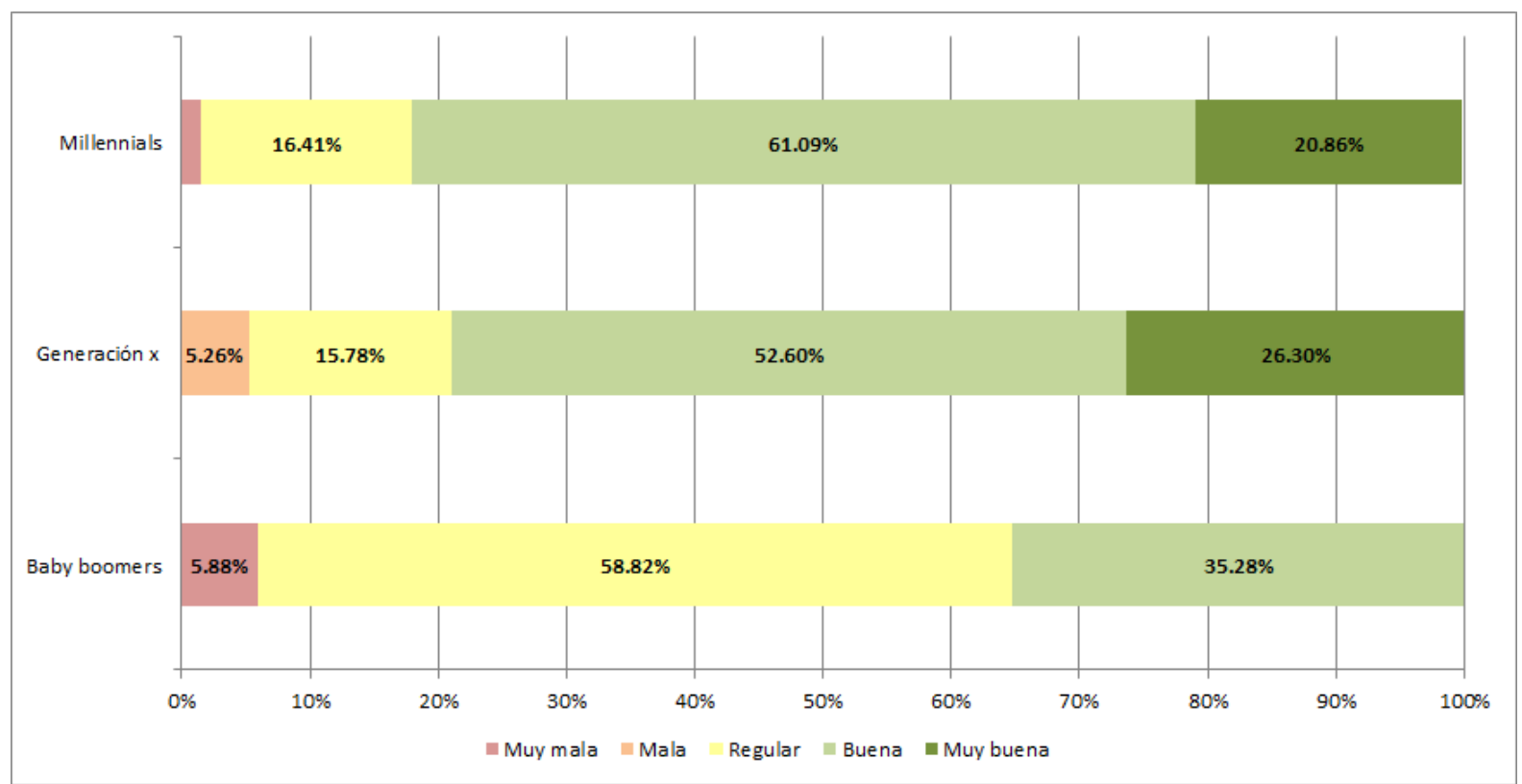

Figura 2. Habilidad tecnológica percibida por generación

La habilidad percibida en el uso de tecnología y los factores de la aceptación de MOOC no mostraron relaciones estadísticamente significativas.

\section{CONCLUSIONES}

En este trabajo, estudiantes de 18 a 60 años inscritos en un programa presencial de inglés reportaron una alta aceptación de los $\mathrm{MOOC}$ que cursaron como complemento a su formación. Sus percepciones de utilidad, facilidad de uso y disfrute de MOOC presentaron correlaciones positivas y significativas. Contrario al planteamiento común que indica que los jóvenes son más propensos a aceptar nuevas tecnologías, se encontró que a mayor edad se incrementa esta aceptación. Esto implica que los adultos mayores están interesados en explorar los MOOC y considerar participar en ellos, y no son tan tecnófobos como se podría suponer.

Así, se enfatiza el valor formativo de los MOOC a lo largo de la vida. Entre las aplicaciones prácticas de este estudio se plantea la posibilidad para el uso de MOOC no sólo como complemento a la educación formal, sino también para el desarrollo de habilidades y conocimientos de personas en una etapa posterior a la universitaria. Se pueden implementar MOOC para atender necesidades de profesionistas, jubilados y más. Sin embargo, dado que la competencia percibida en el uso de tecnología es menor en baby boomers y miembros de la generación $\mathrm{X}$, se recomienda considerar 
apoyo en este ámbito al diseñar o recomendar MOOC. Se pueden incluir recursos que permitan fortalecer la alfabetización digital de quienes así lo requieran. Idealmente, estos serían puestos a prueba con adultos mayores de 50 años para asegurar su pertinencia.

Futuras investigaciones podrían atender algunas de las limitaciones de este estudio. Podrían enfocarse en grupos generacionales más equitativamente distribuidos, evaluar a estudiantes dentro de un mismo MOOC o incluir a nuevas generaciones. Podrían considerar otras variables potencialmente relacionadas con la aceptación de MOOC, como la escolaridad de los usuarios, el aprendizaje percibido y la intención de inscribirse en otros MOOC. Incluso podrían revisar conceptos alternativos a la edad cronológica, como la edad cognitiva. Esto permitiría incrementar el conocimiento existente sobre los perfiles de los usuarios de MOOC, sus necesidades y su participación, y a su vez facilitaría el diseño de cursos más adecuados y efectivos.

\section{REFERENCIAS}

Aboshady, O. A., Radwan, A. E., Eltaweel, A. R., Azzam, A., Aboelnaga, A. A., Hashem, H. A., Darwish, S. Y., Salah, R., Kotb, O. N., Afifi, A. M., Noaman, A. M., Salem, D. S., y Hassouna, A., (2015). Perception and use of massive open online course among medical students in a developing country: multicentre cross-sectional study. BMJ Open, 5(1), 1-9. http://dx.doi.org/10.1136/bmjopen-2014-006804

Aguado Franco, J. C. (2017). ¿Pueden los MOOC favorecer el aprendizaje y hacer disminuir las tasas de abandono universitario? RIED. Revista Iberoamericana de Educación a Distancia, 20(1), 125143. http://dx.doi.org/10.5944/ried.20.1.16684

Angulo Mendoza, G.A., Sauvé, L. y Plante, P. (2017). Adultos mayores y juegos educativos digitales. ¿Qué consideraciones de diseño favorecen su uso? EDUTEC. Revista electrónica de Tecnología Educativa, 62, 104-116. http://dx.doi.org/10.21556/edutec.2017.62.1021

Annabi, C. A. y Wilkins, S. (2016). The use of MOOCs in transnational higher education for accreditation of prior learning, programme delivery, and professional development. International Journal of Educational Management, 30(6), 959-975. https://doi.org/10.1108/IJEM-05-2015-0057

Armellini, A., y Padilla Rodríguez, B. C. (2016). Are Massive Open Online Courses (MOOCs) pedagogically innovative? Journal of Interactive Online Learning, 14(1), 17-28.

Castaño Garrido, C., Maiz Olazabalaga, I., y Garay Ruiz, U., (2015). Percepción de los participantes sobre el aprendizaje en un MOOC. RIED Revista Iberoamericana de Educación a Distancia, 18(2), 197221. https://doi.org/10.5944/ried.18.2.13444

Chung, J. E., Park, N., Wang, H., Fulk, J., y McLaughlin, M. (2010). Age differences in perceptions of online community participation among non-users: An extension of the Technology Acceptance Model. Computers in Human Behavior, 26, 1674-1684. doi:10.1016/j.chb.2010.06.016 
Cole, A. W. y Timmerman, C. E. (2015). What do current college students think about MOOCs? MERLOT Journal of Online Learning and Teaching, 11(2), 188-201.

Davis, F. (1989). Perceived usefulness, perceived ease of use, and user acceptance of information technology. MIS Quarterly, 13, 319-340.

Davis, F., Bagozzi, R., y Warshaw, P. (1992). Extrinsic and intrinsic motivation to use computers in the workplace. Journal of Applied Social Psychology, 22(14), 1111-1132.

Díaz García, I., Cebrián Cifuentes, S., y Fuster Palacios, I. (2016). Las competencias en TIC de estudiantes universitarios del ámbito de la educación y su relación con las estrategias de aprendizaje. Revista Electrónica de Investigación y Evaluación Educativa, 22(1), 1-24. http://dx.doi.org/10.7203/relieve.22.1.8159

Fernández-Ferrer, M. (2017). Democratizando la educación a nivel mundial: ¿ ¿icción o realidad? El papel de los cursos en línea masivos y abiertos. Profesorado. Revista de Currículum y Formación de Profesorado, 21(2), 445-461.

Gallardo Echenique, E. E. (2012). Hablemos de estudiantes digitales y no de nativos digitales. Universitas Tarraconensis. Revista de Ciències de l'Educació, 1(1), 7-21.

González de la Fuente, A., y Carabantes Alarcón, D. (2017). MOOC: medición de satisfacción, fidelización éxito y certificación de la educación digital. RIED Revista Iberoamericana de Educación a Distancia, 20(1), 105-123. http://dx.doi.org/10.5944/ried.20.1.16820

Hauk, N., Hüffmeier, J., y Krumm, S. (2018). Ready to be a silver surfer? A meta-analysis on the relationship between chronological age and technology acceptance. Computers in Human Behavior, 84, 304-319. https://doi.org/10.1016/j.chb.2018.01.020

Hayes, J., Parks, C., McNeilly, S., y Johnson, P. (2018). Boomers to Millennials: Generational Stereotypes at Work in Academic Librarianship. The Journal of Academic Librarianship, 44(6), 845-853. https://doi.org/10.1016/j.acalib.2018.09.011

Ho, A., Chuang, I., Reich, J., Coleman, C., Whitehill, J., Northcutt, C., Williams, J., Hansen, J., Lopez, G., y Petersen, R. (2015). HarvardX and MITX: Two years of open online courses (HarvardX Working Paper No. 10). DOI: 10.2139/ssrn.2586847

Jiménez Cruz, J. R. (2017). Integración de un curso MOOC y de un PLN-PLE en un curso presencial sobre fundamentos de la programación. RED Revista de Educación a Distancia, 53, 1-17. http://dx.doi.org/10.6018/red/53/11

Joo, Y. J., So, H. J., y Kim, N. H. (2018). Examination of relationships among students' self-determination, technology acceptance, satisfaction, and continuance intention to use K-MOOCs. Computers y Education, 122, 260-272. https://doi.org/10.1016/i.compedu.2018.01.003 
Jordan, K. (2015). MOOC Completion Rate: The Data. Recuperado de http://www.katyjordan.com/MOOCproject.html

Khan, I. U., Hameed, Z., Yu, Y., Islam, T., Sheikh, Z., y Khan, S. U. (2018). Predicting the acceptance of MOOCs in a developing country: Application of task-technology fit model, social motivation, and self-determination theory. Telematics and Informatics, 35(4), 964-978. https://doi.org/10.1016/j.tele.2017.09.009

Marangunić, N., y Granić, A. (2013). Technology acceptance model: a literature review from 1986 to 2013. Universal Access in the Information Society, 14(1), 81-95.

Méndez García, C. M. (2013). Diseño e implementación de cursos abiertos masivos en línea (MOOC): expectativas y consideraciones prácticas. RED. Revista de Educación a Distancia, 39, 1-19.

Mulik, S., Yajnik, N., y Godse, M. (2016). Determinants of Acceptance of Massive Open Online Courses. 2016 IEEE Eighth International Conference on Technology for Education. Mumbai, India. (T4E). doi:10.1109/t4e.2016.032

Nordin, N., Norman, H., y Embi, M. A. (2015). Technology acceptance of massive open online courses in Malaysia. Malaysian Journal of Distance Education 17(2), 1-16. http://dx.doi.org/10.21315/mjde2015.17.2.1

Oblinger, D. (2003). Boomers, Gen-Xers, and Millennials: Understanding the "New Students". EDUCAUSE Review, 38(4), 36-45.

Oliver, M., Hernández-Leo, D., y Albó, L. (2015). MOOCs en España. Análisis de la demanda. Panorama actual de los Cursos Masivos Abiertos en Línea en la plataforma Miríada X. Barcelona, España: Universitat Pompeu Rabra. Recuperado de https://repositori.upf.edu/bitstream/handle/10230/25400/MOOCs 2015 Analisidemanda.pd $\underline{f}$

Padilla Rodríguez, B.C., Bird, T., y Conole, G. (2015). Evaluation of Massive Open Online Courses (MOOCs): A Case Study. En T. Bastiaens \& G. Marks (Eds.), Proceedings of Global Learn 2015 (pp. 527-535). Berlín, Alemania: Association for the Advancement of Computing in Education (AACE).

Pappano, L. (2012, 2 de noviembre). The Year of the MOOC. The New York Times. Recuperado de http://www.nytimes.com/2012/11/04/education/edlife/massive-open-online-courses-aremultiplying-at-a-rapid-pace.html

Poy, R., y Gonzales-Aguilar, A. (2014) Factores de éxito de los MOOC: algunas consideraciones críticas. Revista Ibérica de Sistemas y Tecnologías de Información, E1, 105-118. http://dx.doi.org/10.4304/risti.e1.105-118 
Sánchez, M., Kaplan, M., y Bradley, L. (2015). Usando la tecnología para conectar las generaciones: consideraciones sobre forma y función. Comunicar, 45(23), 95-104. http://dx.doi.org/10.3916/C45-2015-10

Shah, D., y Pickard, L. (2019). Massive List of MOOC Providers Around The World. Recuperado de https://www.classcentral.com/report/mooc-providers-list/

Siemens, G. (2013). Massive Open Online Courses: Innovation in Education? En R. McGreal, W. Kinuthia y S. Marshall (Eds.), Open Educational Resources: Innovation, Research and Practice (pp. 5-15). Vancouver: Commonwealth of Learning.

Tao, D., Fu, P., Wang, Y., Zhang, T., y Qu, X. (2019). Key characteristics in designing massive open online courses (MOOCs) for user acceptance: an application of the extended technology acceptance model. Interactive Learning Environments, 1-14. https://doi.org/10.1080/10494820.2019.1695214

Tapia Cortes, C., Navarro Rangel, Y., y de la Serna Tuya, A. S. (2017). El uso de las TIC en las prácticas académicas de los profesores de la Benemérita Universidad Autónoma de Puebla. REDIE Revista Electrónica de Investigación Educativa, 19(3), 14. https://doi.org/10.24320/redie.2017.19.3.1270

Turner, M., Kitchenham, B., Brereton, P., Charters, S., y Budgen, D. (2010). Does the technology acceptance model predict actual use? A systematic literature review. Information and Software Technology, 52(5), 463-479. https://doi.org/10.1016/i.infsof.2009.11.005

Vivar Zurita, H., Vinader Segura, R., y Abuín Vences, N. (2015). Los MOOCs en la enseñanza superior: una tarea pendiente. Estudios sobre el Mensaje Periodístico, 21(1), 629-642. http://dx.doi.org/10.5209/rev ESMP.2015.v21.n1.49115

Wu, B., y Chen, X. (2017). Continuance intention to use MOOCs: Integrating the technology acceptance model (TAM) and task technology fit (TTF) model. Computers in Human Behavior, 67, 221-232. https://doi.org/10.1016/j.chb.2016.10.028

Para citar este artículo:

Rocha Estrada, F. J., Padilla Rodríguez, B. C., y Aguado Franco, J. C. (2020). Diferencias por edad en la aceptación de cursos en línea masivos y abiertos (MOOC). Edutec. Revista Electrónica De Tecnología Educativa, (71), 53-66. https://doi.org/10.21556/edutec.2020.71.1341 